\title{
Electronic-Excitation-Induced Processing in GaSb Compound Nanoparticles
}

\author{
H. Yasuda and H. Mori \\ Research Center for Ultra-High Voltage Electron Microscopy, Osaka University, Osaka 567-0047, Japan \\ Correspondence should be addressed to H. Yasuda, yasuda@uhvem.osaka-u.ac.jp \\ Received 8 April 2010; Revised 2 June 2010; Accepted 25 June 2010 \\ Academic Editor: Bo Zou
}

Copyright ( $) 2011 \mathrm{H}$. Yasuda and H. Mori. This is an open access article distributed under the Creative Commons Attribution License, which permits unrestricted use, distribution, and reproduction in any medium, provided the original work is properly cited.

Temperature dependence of electronic-excitation-induced structural changes in nanoparticles has been studied by in situ transmission electron microscopy. When GaSb nanoparticles kept at $340 \mathrm{~K}$ were excited by $25 \mathrm{keV}$ electrons, the compound transforms to the porous compound or the two-phase structure consisting of an antimony core and a gallium shell with increasing the total electron dose. On the other hand, in GaSb nanoparticles kept at $293 \mathrm{~K}$ the structure remains the original compound phase. It is suggested that such temperature dependence of the structural changes may arise from synergetic behaviors of point defects introduced athermally by the excitation and thermal mobility.

\section{Introduction}

Structural stability in materials under electronic excitation is different from that at the ground state. It is expected that electronic excitation effects on the structural stability will be enhanced in nanoparticles which have high surfaceto-volume ratio and high-atomic mobility. Recently, it was found that when GaSb particles were excited by lowenergy electrons, the compound transforms to a two-phase consisting of an antimony core and a gallium shell with a nanometer-sized void [1-4]. In the present paper, we studied the temperature dependence of such structural changes induced by low-energy electronic excitation in GaSb particles by in situ transmission electron microscopy.

\section{Experimental Procedures}

Preparation of size-controlled GaSb particles was carried out with the use of a double-source evaporator installed in the specimen chamber of an electron microscope. An amorphous carbon film was used as a supporting film and was mounted on a molybdenum grid. Using the evaporator, gallium was first evaporated from one filament to produce gallium particles on the supporting film, and then antimony was evaporated from the other filament onto the same film. The supporting film was kept at ambient temperature during the deposition. Vapour-deposited antimony atoms quickly dissolved into gallium particles to form GaSb (Ga$50 \mathrm{at} \% \mathrm{Sb}$ ) compound particles [5-7]. The particles were then annealed in the microscope at $573 \mathrm{~K}$ for $3.6 \mathrm{ks}$ and were slowly cooled to room temperature in $2.7 \mathrm{ks}$, in an attempt to homogenize the solute concentration in the particles. Electronic excitation experiments and observations were carried out using the same microscope Hitachi $\mathrm{H}$ 7000 TEM operating at an accelerating voltage of $25 \mathrm{kV}$. The electron flux used for excitations was $1.0 \times 10^{20} \mathrm{e} \mathrm{m}^{-2} \mathrm{~s}^{-1}$. The temperature of particles on the supporting films was kept at 293 and $430 \mathrm{~K}$ during the experiments. Structural changes associated with electronic excitations were observed in situ by bright-field images (BFIs) and selected-area electron diffraction patterns (SAEDs).

\section{Results and Discussion}

An example of structural changes in GaSb particles by $25 \mathrm{keV}$ electronic excitation at $430 \mathrm{~K}$ is shown in Figure 1. Figures $1(\mathrm{a})$ and $1\left(\mathrm{a}^{\prime}\right)$ show a BFI of particles with the mean diameter of approximately $20 \mathrm{~nm}$ before excitation 


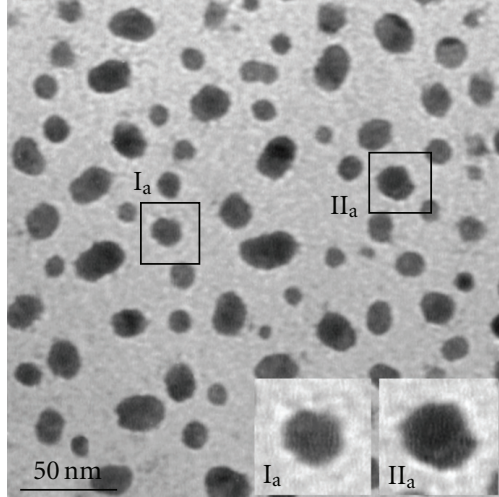

(a)

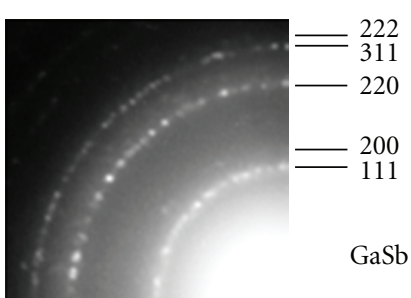

$\left(a^{\prime}\right)$

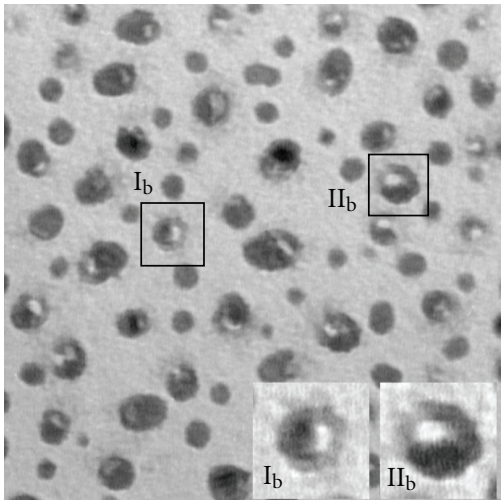

(b)

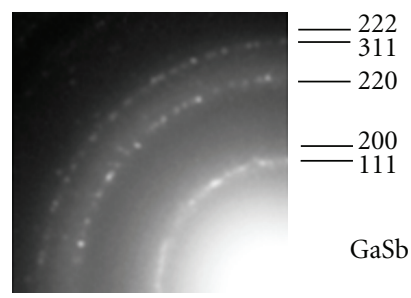

$\left(\mathrm{b}^{\prime}\right)$

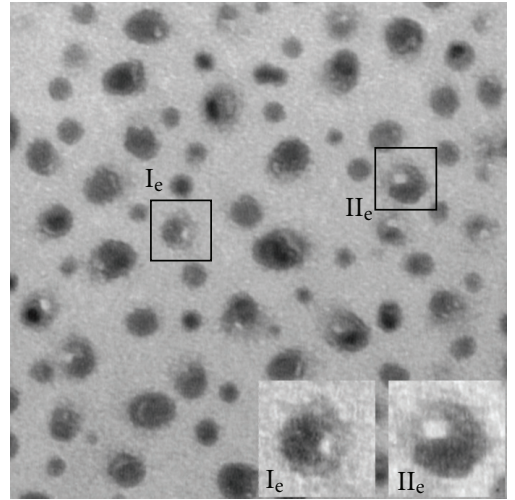

(c)

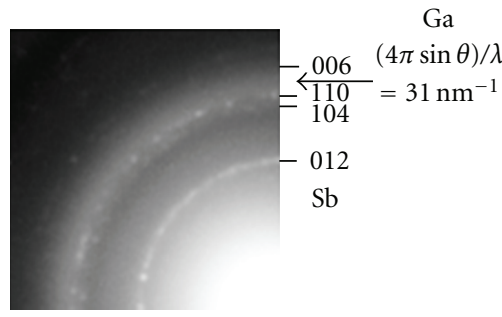

$\left(c^{\prime}\right)$

FIGURE 1: An example of the structural changes in GaSb particles kept at $430 \mathrm{~K}$ by electronic excitation. (a) A BFI and ( $\mathrm{a}^{\prime}$ ) the corresponding SAED before excitation. (b) The same area after excitation for $60 \mathrm{~s}$ and ( $\mathrm{b}^{\prime}$ ) the corresponding SAED. (c) The same area after excitation for $480 \mathrm{~s}$ and $\left(\mathrm{c}^{\prime}\right)$ the corresponding SAED. The parts framed squarely are enlarged in the figures.

and the corresponding SAED, respectively. As indexed in Figure $1\left(\mathrm{a}^{\prime}\right)$, the Debye-Scherrer rings can be consistently indexed as those of $\mathrm{GaSb}$ which has the zincblende structure. The same area after excitation for $60 \mathrm{~s}$ is shown in Figure 1(b). In the interior of the particles after the excitation, there appear voids with bright contrast. As seen from a comparison of the magnified images $I_{a}$ and $I_{a}$ in Figure 1(a) with $\mathrm{I}_{\mathrm{b}}$ and $\mathrm{I}_{\mathrm{b}}$ in Figure $1(\mathrm{~b})$, the diameter of nanoparticles after the excitation increased up to $15 \%$ compared with those before excitation. In the SAED taken after the excitation as shown in Figure 1( $\left.\mathrm{b}^{\prime}\right)$, Debye-Scherrer rings of the zincblende structure are recognized again.

Changes in the lattice constant in GaSb particles are shown as a function of total electron dose in Figure 2. After excitation of the dose of $6.0 \times 10^{21} \mathrm{e} \mathrm{m}^{-2}$, the lattice constant increased up to $1.8 \%$ compared with that before excitation. The lattice constant of GaSb particles under the same excitation condition increased up to about $2.6 \%$ with increasing dose. From the above results, it is suggested that the swelling by void formation in the interior of the individual nanoparticles and the increase of the lattice constant of $\mathrm{GaSb}$ are caused by vacancies and interstitials introduced by electronic excitation, respectively.

The same area after excitation for $480 \mathrm{~s}$ is shown in Figure 1(c). The voids in the individual particles change in the shape and size, as seen from a comparison of the magnified images $\mathrm{I}_{\mathrm{b}}$ and $\mathrm{II}_{\mathrm{b}}$ in Figure $1(\mathrm{~b})$ with $\mathrm{I}_{\mathrm{c}}$ and $\mathrm{II}_{\mathrm{c}}$ in Figure 1(c). In the SAED taken after the excitation as shown in Figure 1 $\left(c^{\prime}\right)$, Debye-Scherrer rings of crystalline antimony are recognized, superimposed on a weak halo ring with the value of the scattering vector $(K=(4 \pi \sin \theta) / \lambda)$ of approximately $31.0 \mathrm{~nm}^{-1}$ which is corresponding to the first halo of liquid gallium. This result indicates that a two-phase mixture consisting of a crystalline antimony core and a liquid gallium shell was formed in the particles. From these results, it has been evident that when GaSb particles kept at $430 \mathrm{~K}$ were excited by $25 \mathrm{keV}$ electrons, two-phase separation takes place via void formation.

In order to see the temperature dependence of the structural changes, experiments in the particles kept at $293 \mathrm{~K}$ were carried out. An example of the behaviors of particles by the same electronic excitation condition as that in Figure 1 is shown in Figure 3. As seen from the comparisons of 3(a) with $3(\mathrm{~b})$ and $3\left(\mathrm{a}^{\prime}\right)$ with $3\left(\mathrm{~b}^{\prime}\right)$, the particle remains unchanged in both the microstructure and SAED after excitation for $60 \mathrm{~s}$. It has been noted here that such a void formation as observed after excitation at $340 \mathrm{~K}$ is absent in the particles kept at $293 \mathrm{~K}$. After excitation for $480 \mathrm{~s}$ as shown in Figures 3(c) and $3\left(\mathrm{c}^{\prime}\right)$, no changes are recognized in the microstructure of the particles and Debye-Scherrer rings in the SAED.

As shown in Figure 2, the lattice constant in GaSb particles kept at $293 \mathrm{~K}$ is not changed also with increasing dose. From the result, it was evident that when approximately $20 \mathrm{~nm}$-sized GaSb particles kept at $293 \mathrm{~K}$ are excited by $25 \mathrm{keV}$ electrons, no structural changes are recognized.

A mechanism of the void formation and phase separation in GaSb nanoparticles will be discussed as follows. In the present case, the final two-hole states in the gallium valence band are formed by the predominant primary K-shell Auger transitions after the excitation. The bonding states under the 


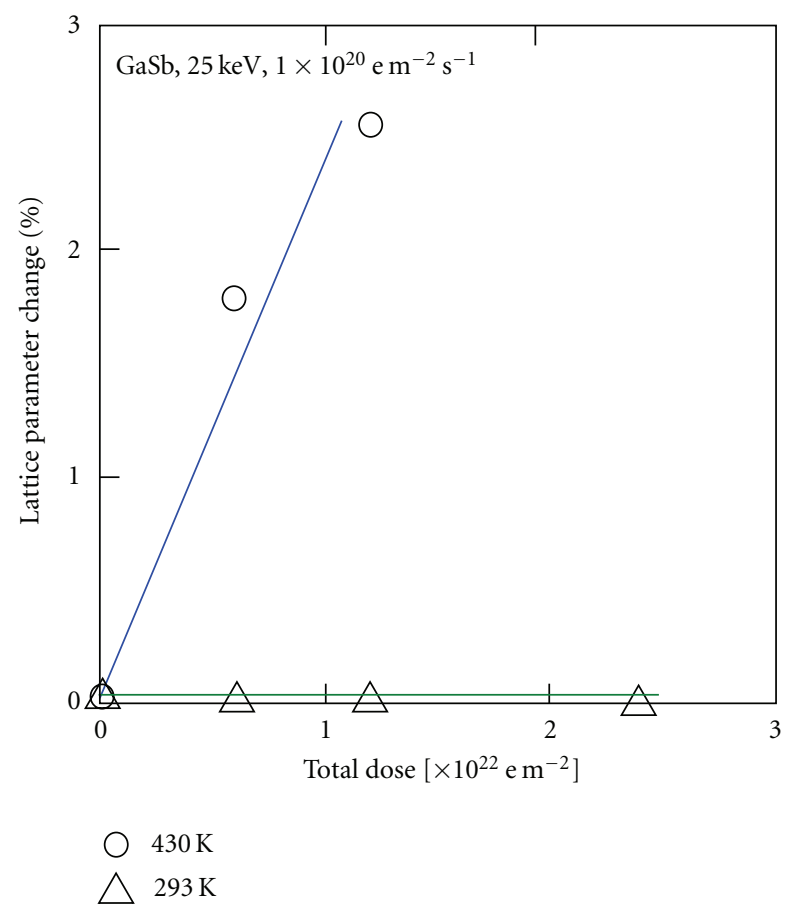

FIGURE 2: Changes in lattice constants of GaSb induced by electronic excitation in nanoparticles as a function of total electron dose.

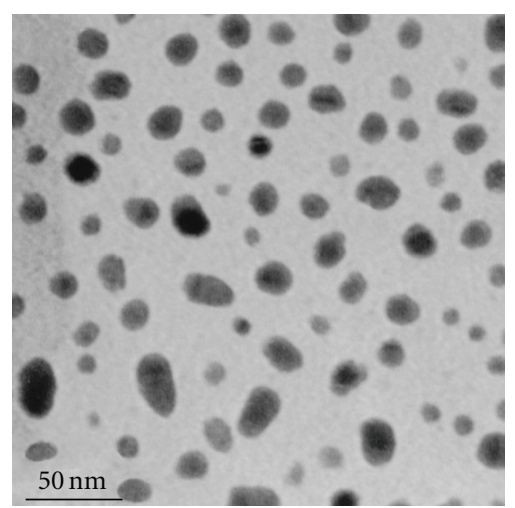

(a)

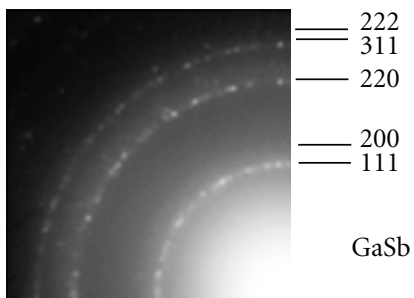

$\left(a^{\prime}\right)$

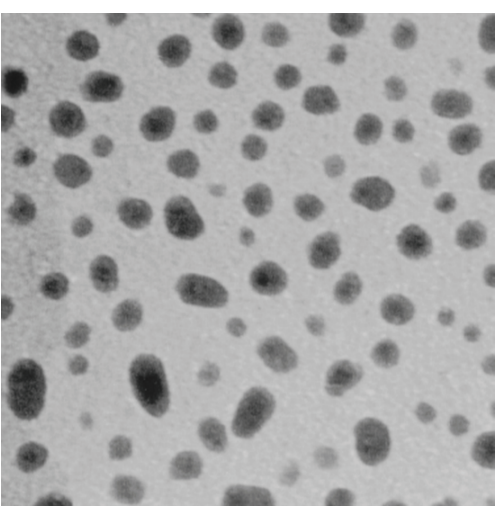

(b)

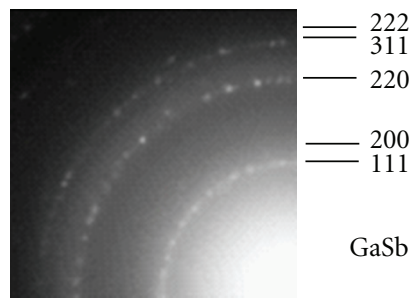

$\left(\mathrm{b}^{\prime}\right)$

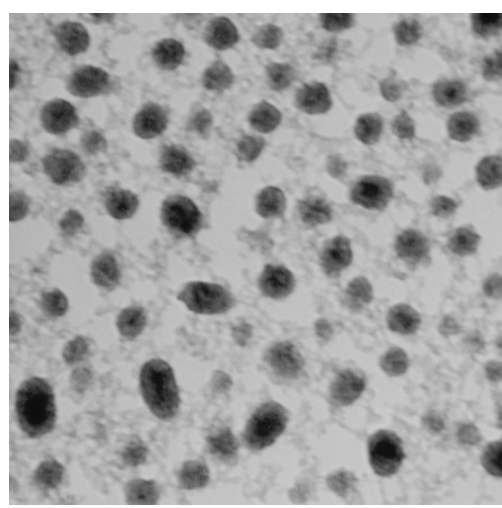

(c)

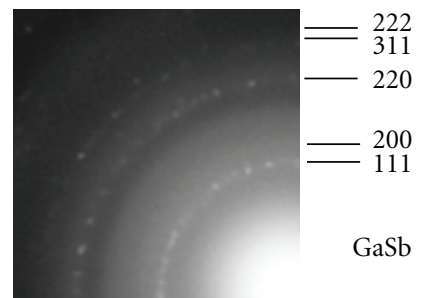

$\left(c^{\prime}\right)$

Figure 3: An example of the structural changes in GaSb particles kept at $293 \mathrm{~K}$ by electronic excitation. (a) A BFI and ( $\mathrm{a}^{\prime}$ ) the corresponding SAED before excitation. (b) The same area after excitation for $60 \mathrm{~s}$ and ( $\mathrm{b}^{\prime}$ ) the corresponding SAED. (c) The same area after excitation for $480 \mathrm{~s}$ and $\left(\mathrm{c}^{\prime}\right)$ the corresponding SAED. 
presence of the two-hole states in gallium atoms become an antibonding. In this first athermal process the bond breaking takes place, and the excess energy accumulated by the relaxation converts directly into the atomic kinetic energy. When the sum of the kinetic energy and thermal energy is larger than the energy barrier for the atomic displacements, the thermally displaced gallium atom can form a vacancy and interstitial in the crystal. In nanoparticles, such an electronic excitation effect becomes remarkably efficient [8].

At elevated temperatures, vacancies and gallium interstitials become mobile and apart from annihilation by recombination, they contribute to the growth of defect clusters. The interstitials and their clusters have a strong compressive strain field; the lattice constant of GaSb becomes large with increasing of the concentration of interstitials and their clusters. This compressive strain field interacts more strongly with a tensile strain field of the surface layer of the particle. The surface layer of the particle will act as a preferential sink for interstitials and their clusters over the whole particles. On the other hand, the capture cross-section of the surface layer for vacancies and their clusters is smaller than that for interstitials and their clusters. The vacancies and their clusters interact weakly with that of the surface layer. In nanoparticles, only the vacancies in the surface layer which is finite in thickness can disappear toward the top of the surface. Consequently, the vacancy concentration in the particle core is higher than that in the surface layer, but interstitial concentration increases toward the surface. Under the condition of vacancy supersaturation in the particle core the vacancy clusters will grow to form a void, and the subsequent surface segregation of interstitial clusters will bring about the separation to the two-phase structure.

In the GaSb particles kept at room temperature, as the energy barrier is too high to jump to the neighbor site, the gallium atoms return to those original positions. Consequently, no lattice constant and structure change after excitation. This mechanism also gives an explanation for temperature dependence of the electronic excitation effect.

\section{Conclusions}

Temperature dependence of electronic-excitation-induced structural changes in nanoparticles has been studied by in situ transmission electron microscopy. When GaSb nanoparticles were excited by $25 \mathrm{keV}$ electrons, the compound transforms to the two-phase structure consisting of an antimony core and a gallium shell with void formations, or remains the original compound phase depending on the temperature. It is suggested that such the temperature dependence of the structural changes may arise from synergetic behaviors of point defects introduced athermally by the excitation and thermal mobility.

\section{Acknowledgments}

This paper was, in part, supported by Priority Assistance for the Formation of Worldwide Renowned Centers of ResearchThe Global COE Program (Project: Center of Excellence for
Advanced Structural and Functional Materials Design) and "Grant-in-Aid for Scientific Research" from the Ministry of Education, Culture, Sports, Science and Technology (MEXT), Japan.

\section{References}

[1] H. Yasuda, H. Mori, and J. G. Lee, "Nonlinear responses of electronic-excitation-induced phase transformations in GaSb nanoparticles," Physical Review Letters, vol. 92, no. 13, Article ID 135501, 4 pages, 2004.

[2] H. Yasuda, H. Mori, and J. G. Lee, "Electron-irradiationinduced phase separation in GaSb nanoparticles," Physical Review B, vol. 70, no. 21, Article ID 214105, 6 pages, 2004.

[3] H. Yasuda, A. Tanaka, H. Usui, H. Mori, and J. G. Lee, "Effect of electron flux on electronic-excitation-induced phase separation in GaSb nanoparticles," European Physical Journal D, vol. 37, no. 2, pp. 231-235, 2006.

[4] H. Yasuda, A. Tanaka, K. Matsumoto, N. Nitta, and H. Mori, "Formation of porous GaSb compound nanoparticles by electronic-excitation- induced vacancy clustering," Physical Review Letters, vol. 100, no. 10, Article ID 105506, 4 pages, 2008.

[5] H. Yasuda and H. Mori, "Spontaneous alloying of zinc atoms into gold clusters and formation of compound clusters," Physical Review Letters, vol. 69, no. 26, pp. 3747-3750, 1992.

[6] H. Yasuda and H. Mori, "Cluster-size dependence of alloying behavior in gold clusters," Zeitschrift für Physik D, vol. 31, no. 1, pp. 131-134, 1994.

[7] H. Yasuda and K. Furuya, "Spontaneous alloying of tin atoms into nanometer-sized gold clusters and phase stability in the resultant alloy clusters," European Physical Journal D, vol. 10, no. 2, pp. 279-283, 2000.

[8] H. Sumi, "Theory on laser sputtering by high-density valenceelectron excitation of semiconductor surfaces," Surface Science, vol. 248, no. 3, pp. 382-410, 1991. 

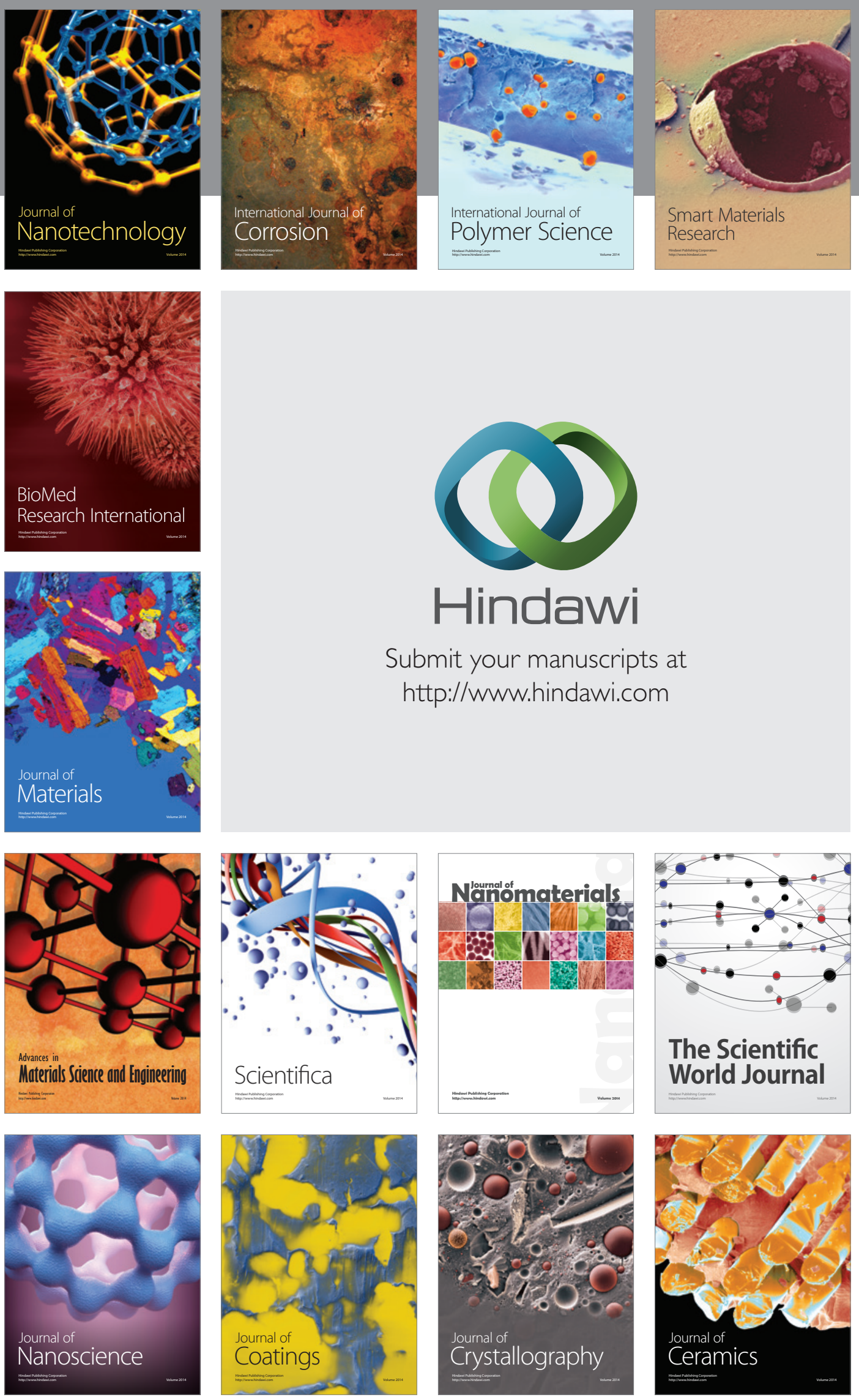

The Scientific World Journal

Submit your manuscripts at

http://www.hindawi.com

\section{World Journal}

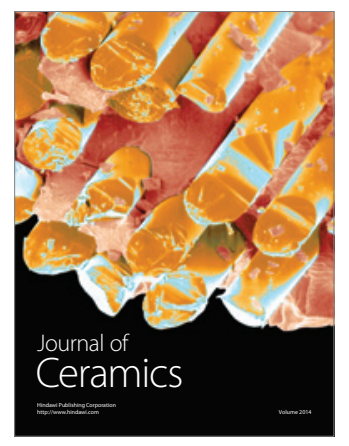

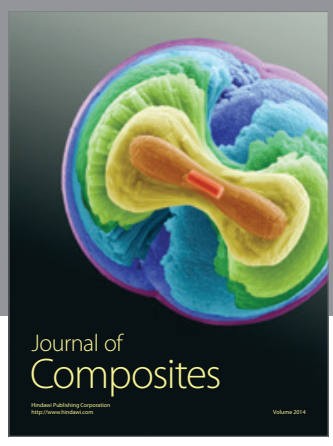
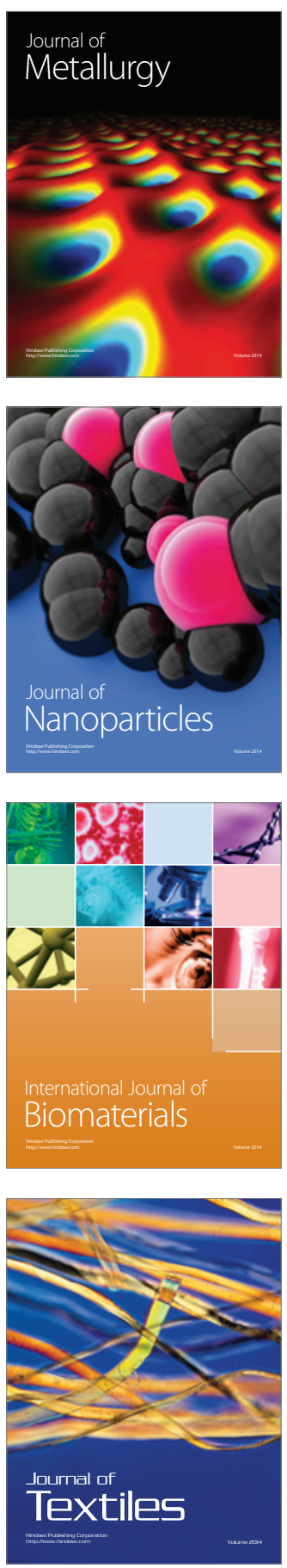